\title{
Early diastolic clicks after the Fontan procedure for double inlet left ventricle: anatomical and physiological correlates
}

\author{
Andrew N Redington, Kit-Yee Chan, Julene S Carvalho, Elliot A Shinebourne
}

\begin{abstract}
$M$ mode echocardiograms and simultaneous phonocardiograms were recorded in four patients with early diastolic clicks on auscultation. All had double inlet left ventricle and had undergone the Fontan procedure with closure of the right atrioventricular valve orifice by an artifical patch. The phonocardiogram confirmed a high frequency sound occurring $60-90 \mathrm{~ms}$ after aortic valve closure and coinciding with the time of maximal excursion of the atrioventricular valve patch towards the ventricular mass. One patient had coexisting congenital complete heart block. The $M$ mode echocardiogram showed "reversed" motion of the patch towards the right atrium during atrial contraction. Doppler flow studies showed that coincident with this motion there was forward flow in the pulmonary artery with augmentation when atrial contraction coincided with ventricular systole.

The early diastolic click in these patients was explained by abrupt cessation of the motion of the atrioventricular valve patch towards the ventricular mass in early diastole. In one patient atrial contraction led to a reversal of this motion and was associated with forward now in the pulmonary artery.
\end{abstract}

For many patients with complex congenital heart disease the only surgical option for long term palliation is the Fontan operation ${ }^{1}$ or one of its modifications. ${ }^{2}$ While most survivors are clinically well after these procedures, abnormal physical signs such as cyanosis, arrhythmia, fluid retention, and heart murmurs are common. Indeed, in a recent review they were found in $62 \%$ of patients. ${ }^{3}$ We report four patients in whom the previously unreported auscultatory sign of an early diastolic click was heard. Simultaneous $M$ mode echocardiography and phonocardiography showed the origin of this sound.

\section{Patients and methods}

Four patients (aged 4-18 years) with loud early diastolic clicking sounds noted on routine auscultation underwent detailed investigation by echophonocardiography. All patients had situs solitus with the usual atrial arrangement. The atrioventricular connection was univentricular, all patients having double inlet ventricle with two atrioventricular valves. All had previously undergone a Fontan type procedure with direct atriopulmonary anastomosis. In two patients a valved homograft was interposed between the right atrium and pulmonary artery. Each patient had the right atrioventricular valve closed by either a single or double layer of Dacron cloth sewn into the floor of the right atrium. The main pulmonary artery was tied off in all.

Cross sectional, $M$ mode, and pulsed wave Doppler echocardiograms with simultaneous electrocardiogram and phonocardiogram were recorded on a Hewlett-Packard 77020A ultrasound system. The phonocardiogram was recorded over the point on the chest where the diastolic click was best heart on auscultation, usually in the fourth left intercostal space close to the sternum. $M$ mode echocardiograms from the left atrioventricular valve, the aortic valve, and the patch over the right atrioventricular valve were recorded with the simultaneous electrocardiogram and phonocardiogram. In one patient, who had congenital complete heart block, an $\mathrm{M}$ mode recording was made of the transverse diameter of the right atrium viewed from a subcostal position. Pulsed wave Doppler flow measurements in the superior and inferior venae cavae (caval veins) and the proximal left pulmonary artery were also recorded in this patient. All recordings were made with a Honeywell (Ecoline 22) strip chart recorder at a paper speed of $100 \mathrm{~mm} / \mathrm{s}$.

\section{Results}

The phonocardiogram confirmed the presence of an early diastolic click in each patient. This was recorded as a high frequency sound occurring $60-90 \mathrm{~ms}$ after the initial high frequency oscillations associated with aortic valve closure, the timing of which was confirmed from the $M$ mode echocardiogram of the aortic valve. There was an inconsistent relation between the sound and left atrioventricular valve motion (fig 1). In each patient, however, the onset of the sound coincided with the time at which the right atrioventricular valve patch had reached its maximal excursion in early ventricular diastole (fig 2).

The patient with congenital complete heart block was studied in further detail. Figure 3 shows a recording of the motion of the right atrioventricular valve patch and its relation to the $P$ wave of the electrocardiogram and subsequent atrial contraction. It is clear from this 
Figure 1 M mode recordings taken from the right atrioventricular valve $(R A V V)$ patch $\{$ top panel) and the left atrioventricular valve (left $A V V$, bottom panel) in the same patient. The early diastolic click (EDC) coincides with the abrupt cessation of movement of the patch towards the ventricle in early diastole and is not related to the motion of the left atrioventricular valve. ECG, electrocardiogram; PCG, phonocardiogram.

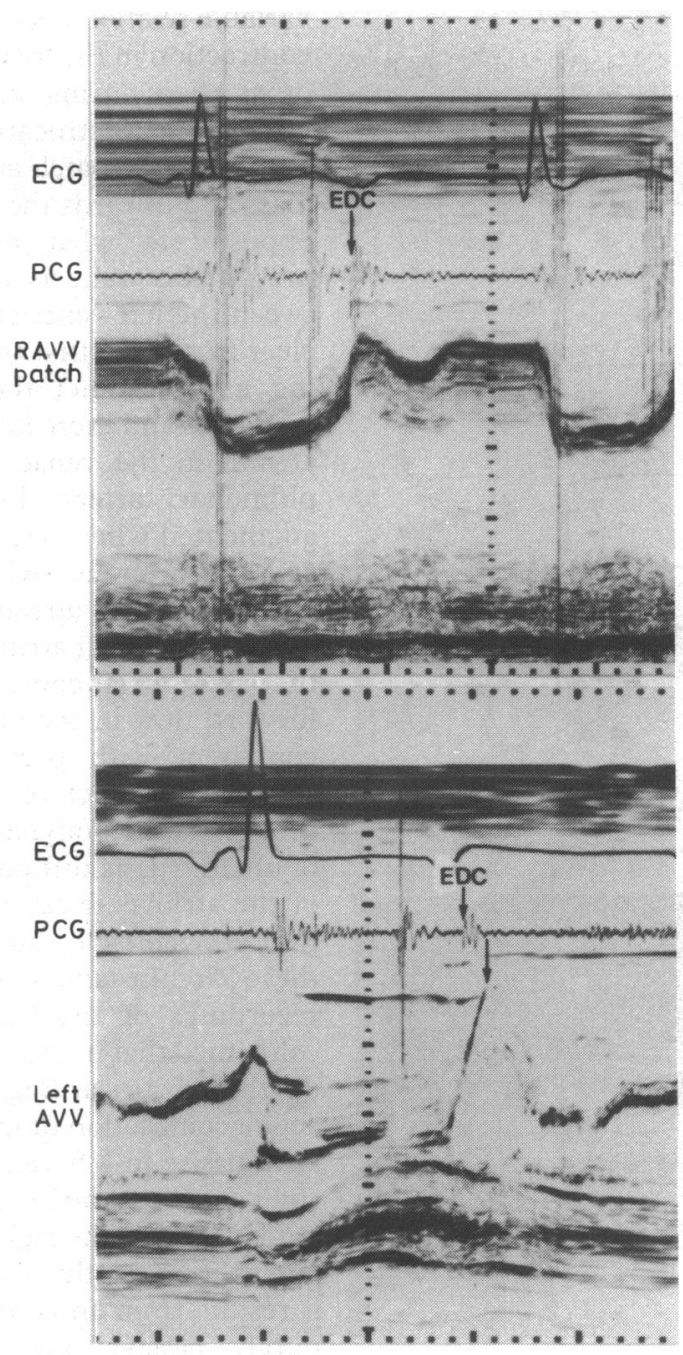

recording that atrial contraction was associated with movement of the patch away from the ventricular mass and towards the right atrium. When this occurred in early diastole there was a prolongation of the interval between aortic valve closure and the early diastolic click (fig 3(A)). This motion presumably reflects a pressure change between ventricle and atrium. Some light was shed on the nature of this pressure change when the atrial dimension and pulsed wave Doppler flow recordings were examined. Figure 4 shows that during atrial
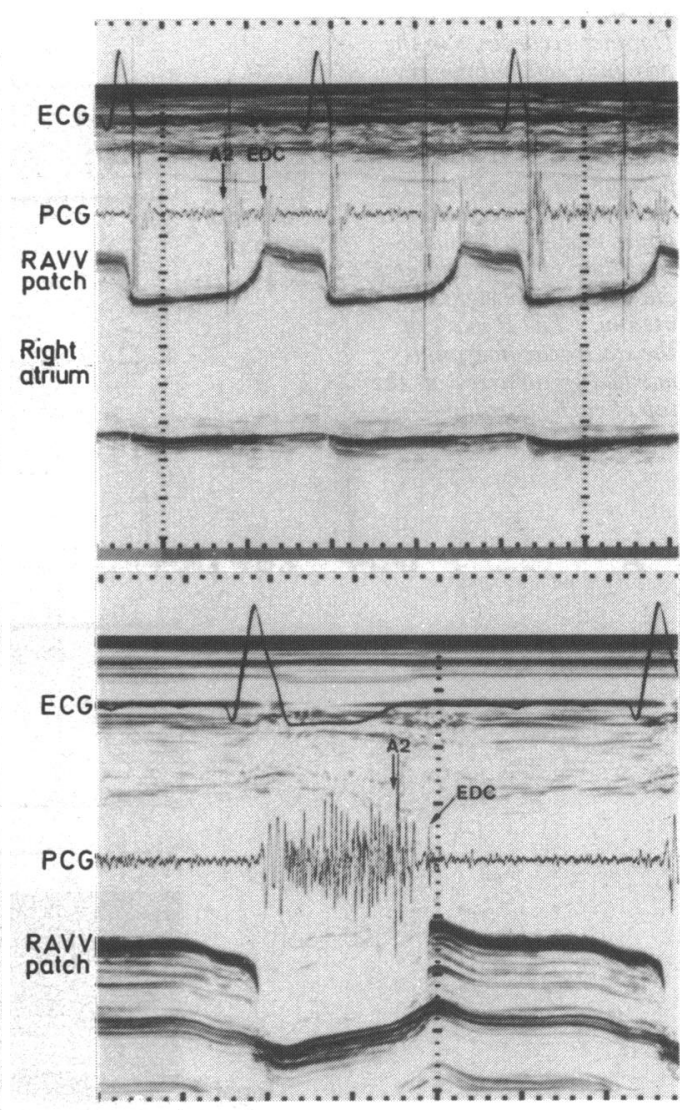

Figure $2 M$ mode recordings of the right

atrioventricular valve (RAVV) patch in two patients. In both the early diastolic click (EDC) occurred approximately $80 \mathrm{~ms}$ after closure of the aortic valve. ECG, electrocardiogram; PCG, phonocardiogram.

systole there was flow away from the atrium, with "reversed" flow in the venae cavae (caval veins) and forward flow in the proximal left pulmonary artery after the $P$ wave of the electrocardiogram. Interestingly, this flow was augmented when the $P$ wave coincided with or was close in timing to the QRS complex. The $M$ mode recording from the right atrium showed that the transverse diameter decreased with atrial systole with the atrial septum moving towards the right atrium after the $P$ wave (fig 4).

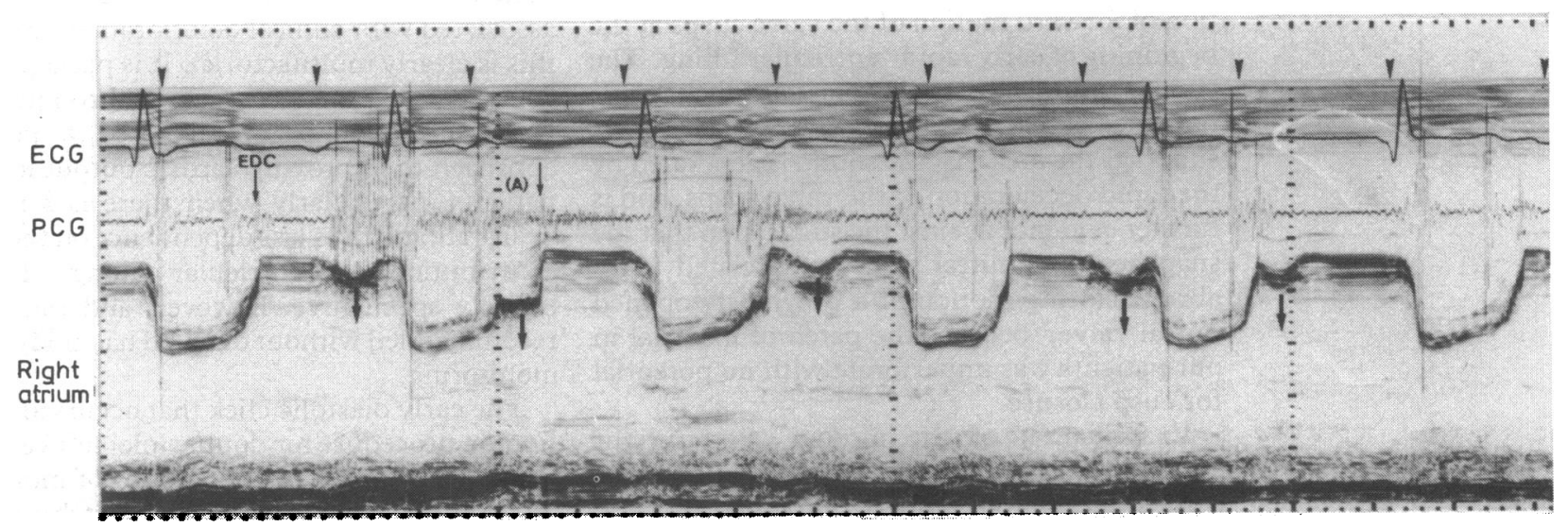

Figure $3 M$ mode recording of the right atrioventricular valve patch in the patient with congenital complete heart block. The $P$ wave of the electrocardiogram (ECG) is marked along the top. When the $P$ wave occurred in diastole the patch moved away from the ventricle towards the right atrium (marked with arrows). The early diastolic click (EDC) was delayed when atrial contraction occurred towards the end of systole ( $A$ ). ECG, electrocardiogram; PCG, phonocardiogram. 
Figure 4 Pulsed wave Doppler recordings in the proximal left pulmonary artery (top panel) and inferior vena cava (IVC, middle panel) from the patient with complete heart block. The bottom panel shows the $M$ mode recording of the transverse diameter of the right atrium. (The $P$ wave of the electrocardiogram is marked by an arrow at the top of each recording-see text for details.)

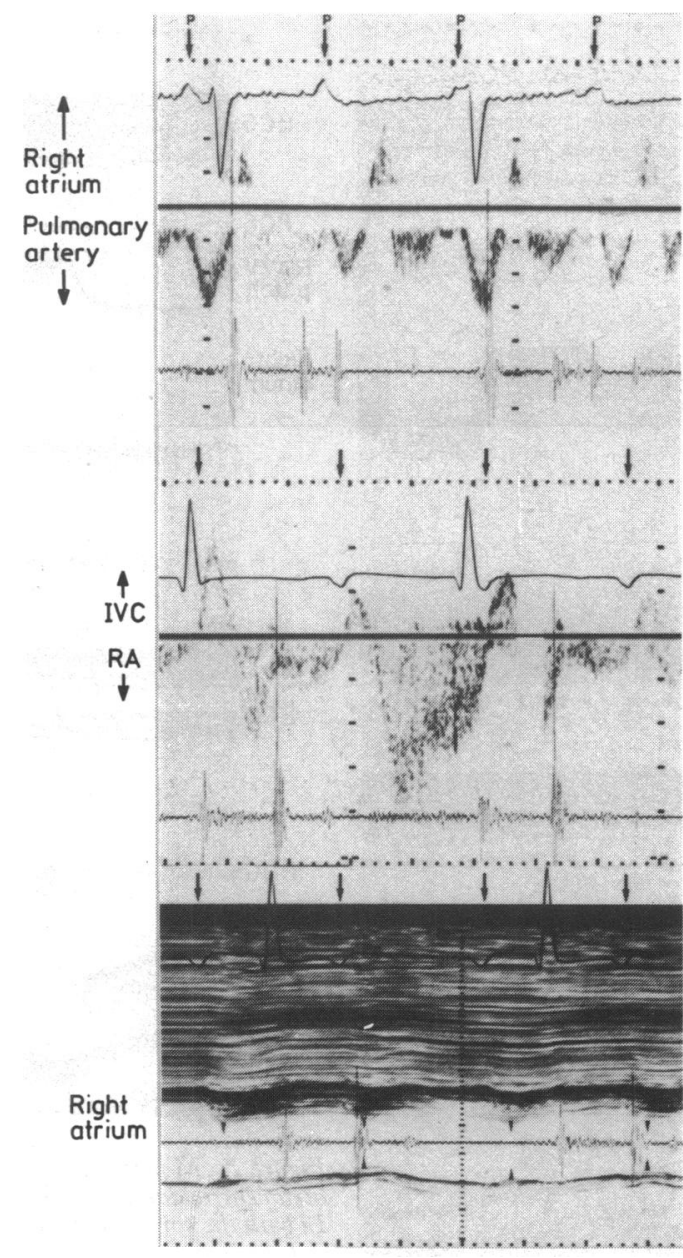

\section{Discussion}

We showed that the early diastolic click heard in patients with double inlet left ventricle after the Fontan procedure coincides with the maximal early diastolic excursion of the synthetic material used to close the right atrioventricular valve orifice. As such, its origins are similar to those of the opening snap heard in patients with mitral or tricuspid stenosis. ${ }^{4-6}$ High frequency opening sounds are frequently heard in patients with Ebstein's anomaly of the tricuspid valve. It was in these patients that $M$ mode recordings with simultaneous phonocardiography were first used to show their origin.? In all these groups of patients the sound has been shown to coincide with the abrupt cessation of forward motion of the valve cusps at the beginning of early rapid ventricular filling. The similar timing of the early diastolic click in our patients, $50-80 \mathrm{~ms}$ after aortic valve closure, supports the idea that the sound is generated by the rapid deceleration of the valve cusps, and is further evidence against the suggestion that the snap heard in mitral stenosis is related to an abrupt closing motion of a previously opened mitral valve ${ }^{8}$ because the patch of material in our patients was imperforate with no potential for cusp closure.

Examination of the motion of the right atrioventricular valve patch in our patient with congenital complete heart block gives us the opportunity to examine the role of atrial contraction in the circulation after the Fontan procedure. As might be expected there was a variable relation between atrial and ventricular contraction in this patient. Figure 3 shows that other than during ventricular systole, the $P$ wave of the electrocardiogram was followed by motion of the patch away from the ventricular mass and towards the right atrium. This is the opposite to what might be expected and presumably reflects a pressure transient between the left ventricle and right atrium. It is clear from fig 4 that the right atrium is emptying at this time; the transverse dimension decreases and there is flow away from the right atrium in the venae cavae (caval veins) and pulmonary artery. Furthermore this flow is augmented when atrial and ventricular systole are synchronous (when the atrioventricular valve patch is already maximally displaced towards the right atrium). It is clear, therefore, that right atrial contraction is contributing to forward flow in the pulmonary artery. This is consistent with previous studies that have shown the effect of atrial contraction ${ }^{910}$ although its haemodynamic importance has been doubted. ${ }^{11}$ It is difficult to explain the motion of the atrioventricular valve patch away from the left ventricle during atrial systole under these circumstances, however. The $M$ mode recordings of the left atrioventricular valve confirmed that it opens shortly after the $P$ wave on the electrocardiogram. It is possible that active contraction of the left atrium leads to an abrupt rise in left ventricular pressure so that right atrial pressure is exceeded, so pushing the patch towards the right atrium. An alternative explanation for this unexpected motion is that it results from an active process rather than a purely passive one. Previous studies have shown that the atrioventricular valve ring follows a similar pattern of motion, ${ }^{12} 13$ that is, with atrial systole it moves towards the atrium, away from the ventricular mass. This is explained by the pattern of insertion of atrial myocardium at right angles to the ring. Thus this motion may be explained if the patch, which is stitched on to or just above the atrioventricular valve ring, moves with this structure during atrial systole.

Irrespective of the mechanism, it seems likely that loss of right atrial contraction in this patient would lead to a fall in cardiac output. It is certainly the case that loss of sinus rhythm in patients after the Fontan procedure is often associated with clinical deterioration, although this is clearly multifactorial. It is perhaps more controversial to suggest that synchronous atrial and ventricular pacing may be a possible approach to improving cardiac output in these patients, particularly when there is a normal heart rate and thus less dependence on the atrial contribution to ventricular filling. This is entirely speculative, however, and cannot be recommended without detailed haemodynamic monitoring.

The early diastolic click that occurs after the Fontan procedure for double inlet left ventricle is related to the abrupt cessation of motion of the right atrioventricular valve patch towards the ventricle. In some patients right atrial contraction may make an important contribution to forward flow in the pulmonary artery, 
which may be augmented when it coincides with ventricular systole.

1 Fontan F, Baudet E. Surgical repair of tricuspid atresia Thorax 1971;26:240-5.

2 Kreutzer GO,Vargas FL, Schlichter AJ, et al. Atriopulmonary anastomosis. J Thorac Cardiovasc Surg 1982;83: ary anasto

3 Leung MP, Benson LN, Smallhorn JF, Williams WG, Trusler GA, Freedom RM. Abnormal cardiac signs after Fontan type of operation: indicators of residua and sequelae. Br Heart J 1989;61:52-8.

4 Wooley CF, Fontana ME, Kilman JW, Ryan JM. Atrial systolic murmur, tricuspid opening snap, and right atrial pressure pulse. Am J Med 1985;78:375-84.

5 Craige EC. On the genesis of heart sounds. Circulation 1976;53:207-9.

6 Craige E, Fortuin NJ. Opening snap in mitral stenosis. Am Heart J 1975;89:128-34.
7 Crews TL, Pridie RB, Benham R, Leatham A. Auscultatory and phonocardiographic findings in Ebstein's anomaly. Correlation of first heart sound with ultrasonic records of tricuspid valve movement. Br Heart J 1972;34:681-7.

8 Rodbard S, Libanoff AJ. The mitral closing snap. Am Heart $J 1972 ; 83: 19-22$.

9 Nakazawa $M$, Nojima $K$, Okuda $H$, et al. Flow dynamics in the main pulmonary artery after the Fontan procedure in patients with tricuspid atresia and single ventricle. Cirpatients with tricuspid atresi

10 DiSessa TG, Child JS, Perloff JK, et al. Systemic venous and pulmonary arterial flow patterns after Fontan's procedure for tricuspid atresi
culation 1984;70:898-902.

11 Nakazawa M, Nakanishi T, Okuda H, et al. Dynamics of right heart flow in patients after Fontan procedure. Circulation 1984;69:306-12.

2 Keren G, Sonnenblick EH, LeJenifel TH. Mitral annulus motion. Circulation 1988;78:621-9.

13 Zaky A, Grabhorn L, Feigenbaum H. Movement of the mitral ring: a study in ultrasonography. Cardiovasc Res $1967 ; 1: 121-31$.

\section{VIEWS FROM THE PAST Myron C Prinzmetal}

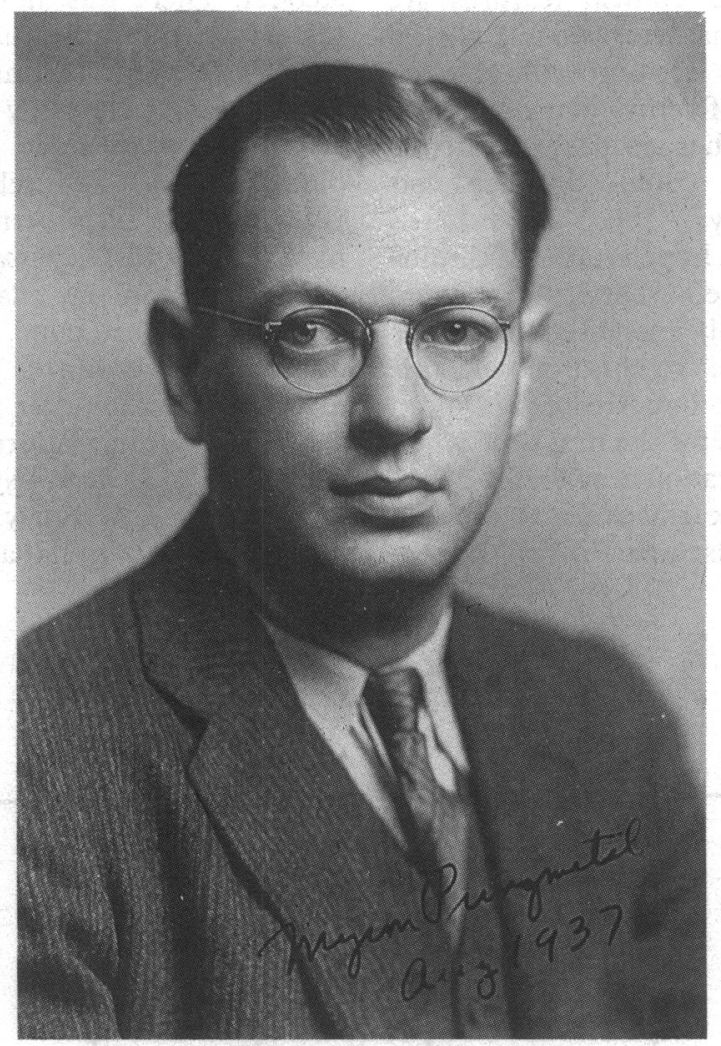

Prinzmetal came to London to work with Lewis before the second world war and continued his studies on arrhythmias even though this was no longer the main thrust of Lewis's work; but Lewis's influence persisted after Prinzmetal had returned to the United States to the Cedars of Lebanon Hospital in Los Angeles. During the war he and Lewis's chief technician, John Honour, exchanged material on the Wolff-Parkinson-White syndrome. While Prinzmetal's book on Auricular arrhythmias (written with several collaborators) is now mainly of historical interest, he is still remembered eponymously for his description of variant angina. His career was ended soon after this discovery by the onset of a debilitating illness which kept him housebound until his death three years ago (Lewis collection). DENNIS M KRIKLER 